\title{
Highlights of Studies in Cardiovascular Disease Prevention Presented at the 2020 American College of Cardiology Annual Scientific Session
}

\author{
Xiaoming Jia ${ }^{1}$ (D) $\cdot$ Mahmoud Al Rifai ${ }^{1} \cdot$ Jing Liu ${ }^{1} \cdot$ Anandita Agarwala ${ }^{2} \cdot$ Martha Gulati $^{3} \cdot$ Salim S. Virani ${ }^{1,4,5}$
}

Published online: 18 June 2020

(C) Springer Science+Business Media, LLC, part of Springer Nature 2020

\begin{abstract}
Purpose of Review The review highlights selected studies related to cardiovascular disease (CVD) prevention that were presented at the American College of Cardiology 2020 Virtual Scientific Session (ACC.20)/World Cardiology Congress (WCC).

Recent Findings The studies reviewed include clinical trials on the efficacy and safety of alirocumab (Study in Participants with Homozygous Familial Hypercholesterolemia [ODYSSEY HoFH]) and evinacumab in the treatment of homozygous familial hypercholesterolemia (HoFH); Evaluating the Efficacy of E-cigarettes for Smoking Cessation (E3); the use of renal denervation in the treatment of hypertension (SPYRAL HTN-OFF MED PIVOTAL); and the assessment of vericiguat in the treatment of heart failure (A Study of Vericiguat in Participants with Heart Failure with Reduce Ejection Fraction [VICTORIA]). In addition, results from the pooled analysis of phase III trials on inclisiran and secondary analysis examining eicosapentaenoic acid levels and cardiovascular outcomes from the Reduction of Cardiovascular Events with Icosapent Ethyl-Intervention Trial (REDUCEIT) were included. Finally, we discuss studies examining the use of polygenic risk score with low density lipoprotein cholesterol (LDL-C) and systolic blood pressure (SBP) on lifetime cardiovascular risk.

Summary The studies presented at the ACC.20/WCC represent notable contributions in the field of CVD prevention.
\end{abstract}

Keywords Cardiovascular disease prevention $\cdot$ Risk factor assessment $\cdot$ ASCVD risk reduction

Xiaoming Jia and Mahmoud Al Rifai contributed equally to this work.

This article is part of the Topical Collection on Reviews and New Research Implications

Salim S. Virani

virani@bcm.edu

1 Section of Cardiology, Baylor College of Medicine, Houston, TX, USA

2 Cardiovascular Division, Washington University School of Medicine in St. Louis, St. Louis, MO, USA

3 Division of Cardiology, University of Arizona College of Medicine-Phoenix, Phoenix, AZ, USA

4 Section of Cardiology, Michael E. DeBakey Veterans Affairs Medical Center, Houston, TX, USA

5 Health Policy, Quality \& Informatics Program, Health Services Research and Development Center for Innovations, Michael E. DeBakey Veterans Affairs Medical Center, 2002 Holcombe Boulevard, Houston, TX 77030, USA

\section{Introduction}

The principle aim of cardiovascular disease (CVD) prevention is in the identification and treatment of risk factors to reduce the burden of CVD morbidity and mortality for individuals and health systems. The American College of Cardiology (ACC) Scientific Sessions 2020 (ACC.20)/World Cardiology Congress (WCC) featured many notable studies in the field of CVD prevention. Due to the coronavirus disease 2019 (COVID-19) outbreak, ACC.20/WCC was held on a virtual platform providing a forum for cardiologists around the world to share and discuss the latest science during an ongoing pandemic. In this review, we highlight findings from 8 studies including late breaking clinical trials and additional select studies relevant to CVD prevention that were presented at ACC.20/WCC (Table 1). As in the past, our aim is to provide succinct summaries of the major findings from these studies and discuss their clinical implications [1-3] .

The studies included clinical trials on the efficacy and safety of alirocumab Study in Participants with Homozygous Familial Hypercholesterolemia [ODYSSEY HoFH]) and evinacumab in the treatment of homozygous familial 
Table 1 Summary of the trials reviewed in this article

\begin{tabular}{|c|c|c|c|c|}
\hline Study & Design & Number of participants & $\begin{array}{l}\text { Follow- } \\
\text { up } \\
\text { duration }\end{array}$ & Principle findings \\
\hline $\begin{array}{l}\text { ODYSSEY } \\
\text { HoFH }\end{array}$ & $\begin{array}{l}\text { Randomized, double-blind, } \\
\text { placebo-controlled }\end{array}$ & $\begin{array}{l}\text { Alirocumab group }(n=45) \\
\text { Placebo group }(n=24)\end{array}$ & 12 weeks & $\begin{array}{l}\text { At } 12 \text { weeks, LDL-C change was }-26.9(4.6) \% \text { from } \\
\text { baseline }(-62.8[14.0] \mathrm{mg} / \mathrm{dL}) \text { in the alirocumab group vs. } \\
8.6(6.3) \% \text { from baseline }(8.9[19.0] \mathrm{mg} / \mathrm{dL}) \text { in the } \\
\text { placebo group. }\end{array}$ \\
\hline HoFH & $\begin{array}{l}\text { Phase 3, randomized, } \\
\text { double-blind, } \\
\text { placebo-controlled }\end{array}$ & $\begin{array}{l}\text { Evinacumab group }(n=43) \\
\text { Placebo group }(n=22)\end{array}$ & 24 weeks & $\begin{array}{l}\text { At } 24 \text { weeks, the evinacumab group showed a LDL-C } \\
\text { change of }-47.1 \text { ( } 4.6) \% \text { from baseline compared with } 1.9 \\
(6.5) \% \text { in the placebo group. }\end{array}$ \\
\hline E3 & Randomized, parallel & $\begin{array}{l}\text { Nicotine E-cigarettes + counseling } \\
\quad(\mathrm{n}=128) \text { Non-nicotine } \\
\text { e-cigarettes + counseling } \\
(n=127)\end{array}$ & 12 weeks & $\begin{array}{l}\text { 1. At } 12 \text { weeks, point prevalence abstinence was } 22 \% \text { in } \\
\text { nicotine e-cigarette group vs. } 17 \% \text { in non-nicotine } \\
\text { e-cigarette group } \\
\text { 2. Relative risk [RR] ( } 95 \% \text { confidence interval [CI]) of the } \\
\text { primary outcome was } 2.4(1.3-4.6) \text { comparing nicotine } \\
\text { e-cigarette vs. counseling and } 1.9(1.0-3.8) \text { comparing } \\
\text { non-nicotine e-cigarette vs. counseling. }\end{array}$ \\
\hline $\begin{array}{l}\text { SPYRAL } \\
\text { HTN-OFF } \\
\text { MED } \\
\text { Pivotal trial }\end{array}$ & Randomized, parallel, sham & $\begin{array}{l}\text { Renal denervation }(n=166) \\
\text { Sham }(n=165)\end{array}$ & 3 months & $\begin{array}{l}\text { 1. Change in } 24-\mathrm{h} \text { systolic blood pressure at } 3 \text { months was } \\
-4.7 \mathrm{mmHg} \text { in the renal denervation group compared } \\
\text { with }-0.6 \mathrm{mmHg} \text { in the sham with a mean }(95 \% \mathrm{CI}) \\
\text { difference of }-4 \mathrm{mmHg}(-6.2 \text { to }-1.8)(p<0.001) \\
\text { 2. Change in office systolic blood pressure at } 3 \text { months, was } \\
-9.2 \mathrm{mmHg} \text { in the renal denervation group compared } \\
\text { with }-2.5 \mathrm{mmHg} \text { in the sham with a mean difference of }- \\
6.6 \mathrm{mmHg}(-9.6 \text { to }-3.5)(\mathrm{p}<0.001)\end{array}$ \\
\hline VICTORIA & $\begin{array}{l}\text { Phase 3, randomized, parallel, } \\
\text { double-blind, } \\
\text { placebo-controlled }\end{array}$ & $\begin{array}{l}\text { Vericiguat group }(n=2526) \\
\text { Placebo group }(n=2524)\end{array}$ & 12 months & $\begin{array}{l}\text { 1. Primary outcome (CV death or heart failure } \\
\text { hospitalization) occurred in } 35.5 \% \text { of the vericiguat } \\
\text { group compared with } 38.5 \% \text { of the placebo group } \\
\text { (hazard ratio [HR] } 0.90, p=0.019) \text {. } \\
\text { 2. HR }(95 \%) \text { for heart failure hospitalization was } 0.90 \\
(0.81-1.00) \text { and for death from cardiovascular causes } \\
\text { was } 0.93(0.81-1.06) \text {. } \\
\text { 3. The composite of death from any cause or hospitalization } \\
\text { for heart failure occurred in } 37.9 \% \text { of the vericiguat } \\
\text { group and } 40.9 \% \text { in the placebo group with a HR ( } 95 \% \\
\text { CI) } 0.90(0.83-0.98) \text {. }\end{array}$ \\
\hline ORION & $\begin{array}{l}\text { Pooled analysis of data from the } \\
\text { ORION } 9 \text {, ORION } 10 \text { and } \\
\text { ORION } 11 \text { phase III trials }\end{array}$ & $\begin{array}{l}\text { Inclisiran group }(n=1833) \\
\text { Placebo group }(n=1827)\end{array}$ & 18 months & $\begin{array}{l}\text { 1. Inclisiran group had significant lowering of LDL-C } \\
\text { compared with placebo (mean difference between groups } \\
\text { was }-55 \%, p<0.0001 \text { at day } 510 \text { and }-52 \%, p<0.0001 \\
\text { when time-averaged from day } 90-540 \text { ). } \\
\text { 2. Similar percentage of patients with at least one TEAE } \\
\text { between the inclisiran and placebo groups ( } 78.0 \% \mathrm{vs} \text {. } \\
77.3 \% \text {, respectively) }\end{array}$ \\
\hline $\begin{array}{l}\text { REDUCE-IT } \\
\text { EPA }\end{array}$ & $\begin{array}{l}\text { Randomized, parallel, } \\
\text { double-blind, } \\
\text { placebo-controlled (secondary } \\
\text { analysis) }\end{array}$ & $N=8179$ & 4.9 years & $\begin{array}{l}\text { 1. No heterogeneity of effect on the primary composite } \\
\text { endpoint with respect to baseline EPA levels stratified by } \\
\text { tertiles ( } p \text { interaction } 0.91 \text { ) } \\
\text { 2. The effect of icosapent ethyl on primary composite } \\
\text { endpoint events is independent of triglycerides and other } \\
\text { biomarkers evaluated except EPA (HR } 1.03,95 \% \text { CI } \\
0.91-1.16 \text { after adjustment). } \\
\text { 3. High EPA levels correspond to higher risk reduction for } \\
\text { primary composite endpoint events ( } p<0.001 \text { for all) }\end{array}$ \\
\hline $\begin{array}{l}\text { Polygenic } \\
\text { Risk Score } \\
\text { (PGS) }\end{array}$ & Mendelian randomization & $N=445,566$ & $\begin{array}{l}\text { Mean } \\
8 \text { years }\end{array}$ & $\begin{array}{l}\text { 1. PGS was significantly associated with major coronary } \\
\text { events (HR } 1.45,95 \% \text { CI } 1.43-1.47 \text { ). } \\
\text { 2. A stepwise increase in risk for coronary events was } \\
\text { further observed across increasing quintiles of PGS } \\
\text { (quintile } 5 \text { vs. quintile 1: HR } 2.77,95 \% \text { CI } 2.65-2.89 \text { ). } \\
\text { 3. Individuals with high PGS and high exposure to LDL-C } \\
\text { and SBP derived the most benefit from LDL-C lowering } \\
\text { (ARR } 13.02 \% \text { ) }\end{array}$ \\
\hline
\end{tabular}

hypercholesterolemia (HoFH); Evaluating the Efficacy of Ecigarettes for Smoking Cessation (E3); the use of renal denervation in the treatment of hypertension (SPYRAL HTN-OFF MED PIVOTAL); and the assessment of 
vericiguat in the treatment of heart failure (A Study of Vericiguat in Participants with Heart Failure with Reduce Ejection Fraction [VICTORIA]). In addition, we included pooled analysis of ORION trials on inclisiran and secondary analysis examining eicosapentaenoic acid levels and cardiovascular outcomes from the Reduction of Cardiovascular Events with Icosapent Ethyl-Intervention Trial (REDUCEIT). Finally, we discuss the used of polygenic risk score with low density lipoprotein cholesterol (LDL-C) and systolic blood pressure (SBP) on lifetime cardiovascular risk.

\section{Monoclonal Antibody Treatments in Homozygous Familial Hypercholesterolemia}

$\mathrm{HoFH}$ is a rare genetic lipid disorder that is characterized by loss of function or impaired function of the LDL receptor (LDLR), which phenotypically presents as extremely elevated LDL-C levels and early onset of atherosclerotic cardiovascular disease (ASCVD) [4]. The most common causal mutation is found within the $L D L R$ gene. Mutations in three other genes within the LDLR pathway, $A P O B$ encoding apolipoprotein $\mathrm{B}$, PCSK9 encoding pro-protein convertase subtilisin/kexin type 9 (PCSK9), and LDLRAP1 encoding LDL receptor adapter protein 1 (LDLRAP), can also lead to the disease $[5,6]$. Genetically, patients with HoFH include those who are true homozygotes but also encompasses compound heterozygotes and double heterozygotes [7]. Due to impairment of the LDLR, most conventional LDL cholesterol lowering medications, which ultimately work by upregulation the LDLR in the liver, are not effective in the treatment of $\mathrm{HoFH}$ [8].

\section{Study Overview: Alirocumab Efficacy and Safety in Adults with Homozygous Familial Hypercholesterolemia (ODYSSEY HoFH)}

Alirocumab is a human monoclonal antibody against PCKS9 that has been shown to significantly lower LDL-C and reduce risk for ASCVD in high-risk secondary prevention patients [9••]. ODYSSEY HoHF was a randomized, double-blind, placebo-controlled trial evaluating the efficacy and safety of alirocumab in patients with HoFH [10]. The study enrolled patients with a diagnosis of HoFH by at least 1 of the following genotypic or clinical criteria: documented homozygous or compound heterozygous mutations in both $L D L R$ alleles; presence of homozygous or compound heterozygous mutations in $A P O B, P C S K 9$, or $L D L R A P 1$; presence of double heterozygous mutations; untreated total cholesterol (TC) $>$ $500 \mathrm{mg} / \mathrm{dL}$ and $\mathrm{TG}<300 \mathrm{mg} / \mathrm{dL}$ and both parents with history of $\mathrm{TC}>250 \mathrm{mg} / \mathrm{dL}$ or cutaneous or tendinous xanthomas before age 10 years. Of note, patients with null mutations in both LDLR alleles were excluded. Other inclusion criteria included age $\geq 18$ years, LDL-C $\geq 70 \mathrm{mg} / \mathrm{dL}$, and being on stable lipid lowering therapy and/or apheresis. Patients were randomized 2:1 to either alirocumab $150 \mathrm{mg}$ IV every 2 weeks or placebo IV every 2 weeks for 12 weeks. The primary efficacy endpoint was percent change (standard error [SE]) from baseline in LDL-C versus placebo at week 12. Secondary endpoints included the proportion of participants with $\geq 30 \%$ and $\geq 50 \%$ reduction in LDL-C at week 12 as well as percent change in TC, apoB, non-high-density lipoprotein cholesterol (non-HDL-C), and lipoprotein (a) (Lp[a]) from baseline to 12 weeks.

Overall, the trial included 69 patients, 45 in the alirocumab group and 24 in the placebo group. The mean baseline LDL-C for the alirocumab and placebo groups were $295.0 \mathrm{mg} / \mathrm{dL}$ and $259.6 \mathrm{mg} / \mathrm{dL}$, respectively. At screening, $97.8 \%$ of participants in the alirocumab group were on a statin, $66.7 \%$ were on statin plus ezetimibe, $15.6 \%$ were on lomitapide, and $13.3 \%$ were on apheresis plus other lipid lowering agents. By comparison, $95.8 \%$ of participants in the placebo group were on a statin, $79.2 \%$ were on statin plus ezetimibe, $12.5 \%$ were on lomitapide, and $16.7 \%$ were on apheresis plus other lipid lowering therapy. At 12 weeks, there was a LDL-C change of -26.9 (4.6)\% from baseline $(-62.8[14.0] \mathrm{mg} / \mathrm{dL})$ in the alirocumab group and $8.6(6.3) \%$ from baseline (8.9 $[19.0] \mathrm{mg} / \mathrm{dL}$ ) in the placebo group. The least squares (LS) mean difference of LDL-C percent change versus placebo at week 12 was $-35.6(7.8) \%, p<0.0001$. There were $57.1 \%$ of participants in the alirocumab group that demonstrated $\geq 30 \%$ reduction in LDL-C at 12 weeks compared to $4.2 \%$ in the placebo group $(p=0.0010)$. Meanwhile, there were $26.7 \%$ of participants in the alirocumab group that demonstrated $\geq$ $50 \%$ reduction in LDL-C at 12 weeks compared with $0 \%$ in the placebo group $(p=0.0017)$. There was a further significant reduction (LS mean [SE]) in TC $(-26.5[6.2] \%, p<0.0001)$, apoB $(-29.8[6.3] \%, p<0.0001)$, non-HDL-C $(-32.9$ $[7.4] \%, p<0.0001)$, and $\mathrm{Lp}$ (a) $(-28.4$ [6.7]\%), $p<0.0001)$. There appeared to be a more consistent effect of alirocumab compared with placebo in patients with compound heterozygous mutations for $L D L R$ and those who were double heterozygotes or heterozygotes ( $L D L R$ mutation + other benign variant). With regard to safety, the percentage of total treatmentemergent adverse events (TEAEs) were numerically similar ( $44.4 \%$ in the alirocumab group and $50.0 \%$ in the placebo group). Injection site reactions (2.2\%), general allergic events $(2.2 \%)$, and diarrhea $(6.7 \%)$ were observed in the alirocumab group but not in the placebo group. There were no serious adverse events, deaths, or discontinuations due to TEAEs.

\section{Study Overview: Evinacumab in Patients with Homozygous Familial Hypercholesterolemia}

Angiopoeitin-like protein 3 (ANGPTL3) is a hepatic secretory protein that inhibits lipoprotein lipase and endothelial lipase, 
enzymes involved in triglyceride and HDL-C metabolism. Mendelian randomization studies have shown that ANGPTL3 is likely casually related to ASCVD [11]. In animal models including LDLR knockouts as well as early human studies of hypercholesterolemia, lowering levels of ANGPTL3 is also associated with reduction in LDL-C, though the mechanism remains unclear [12]. Evinacumab is a human monoclonal antibody directed against the ANGPTL3 protein. Targeting of ANGPTL3 is thought to reduce LDL-C independent of LDLR [13].

This phase 3, randomized, double-blind, placebocontrolled trial assessed the efficacy and safety of evinacumab compared to placebo [14]. Enrolled patients had a diagnosis of HoFH by at least 1 of the following criteria: homozygous mutations in both $L D L R$ alleles; homozygous or compound heterozygous mutations in $A P O B$ or PCSK9; double heterozygous mutations or patients with homozygous LDLRAP1 mutations; untreated TC $>500 \mathrm{mg} / \mathrm{dL}$ and $\mathrm{TG}<300 \mathrm{mg} / \mathrm{dL}$ and both parents with history of TC $>250 \mathrm{mg} / \mathrm{dL}$ or cutaneous or tendinous xanthomas before age 10 years. Included patients also had to be $\geq 12$ years of age, have LDL-C $\geq 70 \mathrm{mg} / \mathrm{dL}$, and be on stable, maximally tolerated lipid-lowering therapy. Included patients were then randomized 2:1 to either evinacumab $15 \mathrm{mg} / \mathrm{kg}$ IV every 4 weeks or placebo IV every 4 weeks for 24 weeks. The primary efficacy endpoint was percent change (standard error [SE]) from baseline in LDL$\mathrm{C}$ versus placebo at week 24 . Key secondary endpoints included absolute change in LDL-C, the proportion of participants with $\geq 30 \%$ and $\geq 50 \%$ reduction in LDL-C at week 24 as well as the proportion of patients who met US apheresis eligibility criteria (LDL $\geq 300 \mathrm{mg} / \mathrm{dL}$ ), and the proportion of patients with LDL-C $<100 \mathrm{mg} / \mathrm{dL}$.

The study included a total of 65 participants, 43 in the evinacumab group and 22 in the placebo group. The mean baseline LDL-C for the evinacumab and placebo groups were $259.5 \mathrm{mg} / \mathrm{dL}$ and $246.5 \mathrm{mg} / \mathrm{dL}$, respectively. At screening, 95.3\% of participants in the evinacumab group were on a statin, $76.7 \%$ were on ezetimibe, $79.1 \%$ were on a PCSK9 inhibitor, $48.8 \%$ were on statin, ezetimibe plus PCSK9 inhibitor, $25.6 \%$ were on lomitapide, and $32.6 \%$ received apheresis. In the placebo group, $90.9 \%$ were on a statin, $72.7 \%$ were on ezetimibe, $72.7 \%$ were on a PCSK9 inhibitor, $36.4 \%$ were on statin, ezetimibe plus PCSK9 inhibitor, $13.6 \%$ were on lomitapide, and $36.4 \%$ received apheresis. At 24 weeks, the evinacumab group showed a LDL-C change of -47.1 (4.6)\% from baseline compared with $1.9(6.5) \%$ in the placebo group. The LS mean difference for LDL-C percent change versus placebo was $-49.0(8.0) \%, p<0.0001$. There did not appear to be a significant difference of effect in participants with null/null mutations compared with those with not null/null mutations. The absolute change in LDL-C was -134.7 (12.4) $\mathrm{mg} / \mathrm{dL}$ in the evinacumab group versus $-2.6(17.6)$ $\mathrm{mg} / \mathrm{dL}$ in the placebo group with a LS mean difference of -
$132.1(21.5) \mathrm{mg} / \mathrm{dL}, p<0.0001)$. When evinacumab was compared with placebo, $83.7 \%$ vs. $18.2 \%$ demonstrated $\geq$ $30 \%$ reduction in LDL-C at 24 weeks $(p<0.0001) ; 55.8 \%$ vs. $4.5 \%$ demonstrated $\geq 50 \%$ reduction in LDL-C at 24 weeks $(p=0.003) ; 7.0 \%$ vs. $22.7 \%$ met US apheresis eligibility criteria at 24 weeks ( $p=0.085$ ); $46.5 \%$ vs. $22.7 \%$ had LDL$\mathrm{C}<100 \mathrm{mg} / \mathrm{dL}$ at 24 weeks $(p=0.020)$. There was a further significant reduction (LS mean) in TC $(-48.4 \%, p<0.0001)$, apoB $(-36.9 \%, p<0.0001)$, non-HDL-C $(-51.7 \%$, $p<0.0001)$, and triglycerides $(-50.4 \%, p<0.0001)$. There was not a significant change in $\mathrm{Lp}$ (a) between the evinacumab and placebo groups at 24 weeks $(-1.9 \%, p=0.7906)$. With regard to safety, there were numerically less TEAEs in the evinacumab compared with the placebo group $(65.9 \%$ vs. $81.0 \%$ ). However, there were numerically more, though rare, SAEs in the evinacumab group compared with placebo $(4.5 \%$ vs. $0 \%$ ).

\section{Clinical Implications}

HoFH patients are at very high risk for the development of ASCVD due to cumulative exposure to severely elevated LDL-C levels starting in early age. Moreover, HoFH is a difficult lipid disorder to treat given modest response to traditional lipid lowering agents. The above studies suggest the PCSK9 inhibitor alirocumab has some effect on LDL-C, which is consistent with previous studies in $\mathrm{HoFH}$ with evolocumab [15•]. However, the response to treatment appeared more robust and consistent in patients who were compound or double heterozygotes compared with those who were homozygotes for pathogenic mutations. In addition, null/null homozygous patients were excluded from ODYSSEY-HoFH. Evinacumab, which likely acts independently of LDLR pathway, appears to be a more potent LDL-C agent in patients with $\mathrm{HoFH}$, even in those with null/null LDLR mutations. The results from the evinacumab study, while promising, should be viewed with caution as more data is needed to ascertain its long-term efficacy, safety, and tolerability. Finally, these studies underscore the potential role of genetic testing in $\mathrm{HoFH}$, which may ultimately guide therapy.

\section{Efficacy and Safety of E-Cigarettes for Smoking Cessation-E3}

\section{Study Overview}

The E3 trial was a randomized trial of active smokers who were motivated to quit smoking, comparing the use of nicotine e-cigarettes plus counseling $(n=128)$ vs. non-nicotine e-cigarettes plus counseling $(n=127)$ versus counseling alone $(n=$ 121) [16]. The primary outcome was point prevalence abstinence at 12 weeks, which is a commonly used outcome 
measure in smoking cessation trials. At 12 weeks, participants were asked whether they had smoked 1 puff of cigarettes in the previous week, which was then confirmed by carbon monoxide testing (a negative test or abstinence was defined as achieving a reading of less than $10 \mathrm{ppm}$ ). Secondary outcomes included number of cigarettes smoked per day and continuous abstinence.

The trial included a total of 376 active smokers of mean age 52 years, $47 \%$ female, mean smoking duration 35 years, and mean number of cigarettes per day of 21 . At 12 weeks, point prevalence abstinence was $22 \%$ in the nicotine e-cigarette group compared with $17 \%$ in the non-nicotine e-cigarette group, and $9 \%$ in the counseling alone group. The relative risk [RR] $(95 \%$ confidence interval $[\mathrm{CI}])$ of the primary outcome was 2.4 (1.3-4.6) comparing nicotine e-cigarette vs. counseling and 1.9 (1.0-3.8) comparing non-nicotine e-cigarette vs. counseling. The number of cigarettes smoked per day was 8 in the nicotine e-cigarette group, 10 in the non-nicotine ecigarette group, and 14 in the counseling alone group. The mean $(95 \% \mathrm{CI})$ difference in number of cigarettes per day was $-5.7(-8.0$ to -3.3$)$ comparing nicotine e-cigarette vs. counseling and $-3.6(-6.3$ to -1.0$)$ comparing nonnicotine e-cigarette vs. counseling. Continuous abstinence was $5 \%$ in the nicotine e-cigarette group versus $3 \%$ in the non-nicotine e-cigarette group versus $1 \%$ in the counseling alone group. This translated to a RR $(95 \% \mathrm{CI})$ of 5.7 (0.7-46.4) comparing nicotine e-cigarette vs. counseling and $3.8(0.4-33.6)$ comparing non-nicotine e-cigarette vs. counseling.

\section{Clinical Implications}

In E3, nicotine e-cigarettes plus counseling was superior to counseling alone for smoking cessation. Non-nicotine e-cigarettes plus counseling was also more effective compared with counseling alone though its effects on cessation were modest. This trial demonstrates the efficacy of e-cigarettes as a tool for smoking cessation compared with counseling alone. However, a limitation of this study is that it did not include nicotine patch or varenicline as a comparator arm. Additionally, this study only assessed cessation at 12 weeks and not long-term smoking cessation. Safety (both short-term and long-term) remains unknown. At this point, e-cigarettes cannot be recommended as first line therapy for the smoking cessation. The long-term health effects of e-cigarettes remain largely unknown and tobacco use patterns (e.g. dual use of cigarettes and e-cigarettes) with long-term vaping need to be established. While the results of E3 hold promise for ecigarettes as a smoking cessation aid, more data on their safety and long-term efficacy is needed to make the case for their routine use for this purpose.

\section{SPYRAL HTN OFF MED: Renal Denervation Potential Option for Patients with Untreated Hypertension}

\section{Study Overview}

The SPYRAL HTN-OFF MED PIVOTAL trial evaluated the efficacy of renal denervation for reducing blood pressure among patients with uncontrolled hypertension [17]. This randomized controlled trial comparing renal denervation versus a sham procedure consisted of patients who were not on antihypertensive medication or those in whom drug therapy could be discontinued. Before randomization, patients were required to discontinue any antihypertensive agents. Thresholds for blood pressure included were office systolic blood pressure (SBP) $\geq$ 150 and $<180 \mathrm{mmHg}$, diastolic blood pressure (SBP) $\geq$ $90 \mathrm{mmHg}$, or systolic 24-h mean ambulatory blood pressure monitoring $\geq 140$ and $<170 \mathrm{mmHg}$. Patients were excluded if they had unclear renal artery anatomy, estimated glomerular filtration rate $<45 \mathrm{ml} / \mathrm{min} / 1.73 \mathrm{~m} 2$, type 1 diabetes or type 2 diabetes with glycated hemoglobin A1c $>8.0 \%$, or secondary hypertension. The primary efficacy endpoints were change in 24-h SBP at 3 months and change in office SBP at 3 months.

A total of 331 patients (166 in the denervation group and 165 in the sham group) were included in the trial with mean age 52 years, $35 \%$ female, and $3.6 \%$ with diabetes mellitus. Change in 24-h SBP at 3 months was $-4.7 \mathrm{mmHg}$ in the renal denervation group compared with $-0.6 \mathrm{mmHg}$ in the sham group with a mean $(95 \% \mathrm{CI})$ difference of $-4 \mathrm{mmHg}(-6.2$ to $-1.8)(p<0.001)$. Differences in 24-h SBP were maintained during both daytime and nighttime. Furthermore, change in office SBP at 3 months, was $-9.2 \mathrm{mmHg}$ in the renal denervation group compared with $-2.5 \mathrm{mmHg}$ in the sham group with a mean difference of $-6.6 \mathrm{mmHg}(-9.6$ to -3.5$)$ $(p<0.001)$. These results were consistent across prespecified subgroups. There were no major device- or procedure-related safety events through the end of the trial at 3 months.

\section{Clinical Implications}

The SPYRAL HTN-OFF MED showed that renal artery denervation compared with sham can safely reduce blood pressure among patients with uncontrolled hypertension in the absence of antihypertensive medications. These effects persisted after 3 months of the procedure. However, a substantial number of patients were started on antihypertensive therapy due to systolic blood pressure $>180 \mathrm{mmHg}$ or for safety reasons in $9.6 \%$ in the renal denervation group and $17 \%$ in the sham groups respectively $(p=0.049)$. Previously, SYMPLICITY HTN-3 examined the effect of renal denervation on patients with resistant hypertension. In this randomized trial mean $( \pm \mathrm{SD})$ change in $\mathrm{SBP}$ at 6 months was -14.13 $\pm 23.93 \mathrm{mmHg}$ in the denervation group compared with - 
$11.74 \pm 25.94 \mathrm{mmHg}$ in the sham-procedure group but the difference was not statistically significant $(p=0.98)$. One potential reason for the difference in results between the two trials is that patients in SPYRAL were required to be off antihypertensive medications, whereas patients in SIMPLICITY were not. Additional long-term data are required to determine what proportion of patients can remain off antihypertensive treatment long-term and whether renal artery denervation has efficacy in patients on antihypertensive medications.

\section{Vericiguat in Patients with Heart Failure and Reduced Ejection Fraction-VICTORIA}

\section{Study Overview}

The VICTORIA trial examined the effect of vericiguat, a novel oral soluble guanylate cyclase stimulator, among patients with heart failure with reduced ejection fraction ( $\mathrm{HFrEF})$ who had recently been hospitalized or had received intravenous diuretic therapy [18]. This phase 3, randomized, double-blind, placebo-controlled trial of 5050 patients with chronic heart failure (New York Heart Association class II to IV) and with an ejection fraction of less than $45 \%$, compared vericiguat (target dose, $10 \mathrm{mg}$ once daily) versus placebo, in addition to guideline-directed medical therapy (GDMT). The primary outcome was a composite of cardiovascular mortality or first hospitalization for heart failure.

The mean age of trial participants was 67 years, and $24 \%$ were women. About $2 / 3$ of patients at randomization were enrolled within 3 months of their index hospitalization for heart failure, $40 \%$ were classified as having NYHA class III heart failure, and the mean ejection fraction was $29 \%$. There was adequate use of guideline-directed medical therapy in both arms (73\% angiotensin converting enzyme inhibitor or angiotensin receptor blocker, $93 \%$ beta blocker, $70 \%$ mineralocorticoid antagonist, and 14\% angiotensin receptorneprilysin inhibitor). Of note, the study authors anticipated an event rate of $11 \%$ at 12 months among patients in the placebo group but there was a higher than anticipated event rate of $17.5 \%$. Therefore, interim futility and efficacy analyses were planned but did not occur. Over a median of 10.8 months, the primary outcome occurred in $35.5 \%$ in the vericiguat group and in $38.5 \%$ placebo group with a hazard ratio (HR) (95\% confidence interval $[\mathrm{CI}]) 0.90(0.82-0.98), p=0.02$. The corresponding HR $(95 \% \mathrm{CI})$ for heart failure hospitalization was $0.90(0.81-1.00)$ and for cardiovascular mortality was $0.93(0.81-1.06)$. The composite of all-cause mortality or hospitalization for heart failure occurred in $37.9 \%$ of the vericiguat group and $40.9 \%$ in the placebo group with a HR (95\% CI) $0.90(0.83-0.98), p=0.02$. The effect of vericiguat on the primary outcome remained consistent across most prespecified subgroups. With regard to safety endpoints, symptomatic hypotension occurred in $9.1 \%$ of the patients in the vericiguat group and in $7.9 \%$ of the patients in the placebo group ( $p=0.12$ ), and syncope occurred in $4.0 \%$ of the patients in the vericiguat group versus $3.5 \%$ of the patients in the placebo group $(p=0.30)$.

\section{Clinical Implications}

Among high-risk patients with heart failure and reduced ejection fraction, the incidence of death from cardiovascular causes or hospitalization for heart failure was lower among those who received vericiguat compared with placebo when added to GDMT. This trial examines a unique subset of heart failure patients who are at particularly high risk of recurrent hospitalization or cardiovascular mortality given that the study population included patients with recent hospitalization for heart failure in the past 6 months $(0-3$ or 3-6 months before randomization) or those who received intravenous diuretic therapy, without hospitalization in the previous 3 months. The addition of vericiguat to GDMT was beneficial for reducing the composite primary outcome of death from cardiovascular causes or first hospitalization for heart failure with a number needed to treat of approximately 24 . Further studies are required to determine the long-term benefits and safety of vericiguat. Cost-benefit analyses are also needed to determine cost savings with use of this novel drug.

\section{ORION: a Pooled Analysis of Phase III Studies of Inclisiran}

PCSK9 inhibitors are potent therapeutic agents for the lowering of LDL-C and reduction of ASCVD risk. Currently commercially available PCSK9 inhibitors are human monoclonal antibodies targeting the PCSK9 protein. Inclisiran is a novel, small interfering ribonucleic acid (siRNA) agent that targets the messenger RNA (mRNA), which acts by silencing PCSK 9 translation and in turn lowers LDL-C [19॰].

\section{Study Overview}

This study was a pooled analysis of data from the ORION 9, ORION 10, and ORION 11 phase III trials, which assessed the efficacy and safety of inclisiran in reducing LDL-C [19•, 20, 21 in patients $\geq 18$ years of age on maximally tolerated statin therapy \pm ezetimibe. In ORION 9, patients with heterozygous $\mathrm{FH}(\mathrm{HeFH})$ diagnosed by genetic testing and/or Simon Broome criteria, who were on a stable low-fat diet and with LDL-C $\geq 100 \mathrm{mg} / \mathrm{dL}$ were included. In ORION 10, patients with ASCVD with LDL-C $\geq 70 \mathrm{mg} / \mathrm{dL}$ were included. In ORION 11, individuals with ASCVD with LDL-C $\geq 70 \mathrm{mg} /$ $\mathrm{dL}$ or ASCVD risk equivalents (type 2 diabetes, 10-year risk $\geq 20 \%$, or $\mathrm{HeFH}$ ) with LDL-C $\geq 100 \mathrm{mg} / \mathrm{dL}$ were included. 
The primary efficacy endpoint was percentage LDL-C change vs. placebo while the safety endpoints were TEAEs and safety laboratory parameters.

The pooled analysis included 3660 patients (1833 in the inclisiran group and 1827 in the placebo group). The mean baseline LDL-C in the inclisiran group was $112 \mathrm{mg} / \mathrm{dL}$ and in the placebo groups was $111 \mathrm{mg} / \mathrm{dL}$. For both the inclisiran and placebo groups at baseline, $92 \%$ of participants were on a statin of which $74 \%$ were on a high-intensity statin. Over 18 months, the inclisiran group had significant reduction in LDL-C when compared with placebo with a mean difference of $-55 \%, p<0.0001$ at day 510 and $-52 \%, p<0.0001$ when time-averaged from day 90 to 540 . The analysis showed greater odds of achieving LDL-C thresholds of $<100 \mathrm{mg} / \mathrm{dL},<$ $70 \mathrm{mg} / \mathrm{dL},<50 \mathrm{mg} / \mathrm{dL}$, and $<25 \mathrm{mg} / \mathrm{dL}$ in patients receiving statin + inclisiran compared with those on statin alone. There was a significant reduction (mean percentage change from baseline compared to placebo) in other parameters including PCSK9 (-83\%), TC (-32\%), non-HDL-C (-46\%), apoB ($42 \%$ ), and $\mathrm{Lp}$ (a) $(-20 \%)$ (all $p$ values $<0.0001)$. There was a more robust reduction of LDL-C across pre-specified subgroups, though $p$ interaction were not provided.

With respect to safety outcomes, there was numerically similar percentage of patients with at least one TEAE between the inclisiran and placebo groups $(78.0 \%$ vs. $77.3 \%$, respectively). Individual TEAEs also appeared to be numerically similar between groups except for a higher percent of injection site TEAEs in the inclisiran group compared with placebo $(5.0 \%$ vs. $0.7 \%)$. Significant liver, kidney, muscle, and hematologic laboratory abnormalities were rare $($ all $<1 \%)$ and were numerically similar between the inclisiran group compared with placebo.

\section{Clinical Implication}

Pooled analysis of the three ORION trials demonstrated sustained efficacy and favorable safety profile of inclisiran in lowering LDL-C on a background of maximally tolerated statin therapy. As inclisiran is available as an intravenous formulation that can be administered every 6 months, it provides an added potential benefit of improved medication adherence. However, a cardiovascular outcomes trial is needed to evaluate whether administration of inclisiran in select patients can also lower risk for ASCVD events.

\section{EPA Levels and Cardiovascular Outcomes in the Reduction of Cardiovascular Events with Icosapent Ethyl-Intervention (REDUCE-IT) Trial}

The REDUCE-IT trial was a randomized placebo-controlled trial of 8179 participants, assessing the efficacy and safety of highly purified EPA, icosapent ethyl at $2 \mathrm{~g}$ twice daily, in the reduction of risk for ASCVD events. The study included individuals $\geq 45$ years of age with either established ASCVD or diabetes mellitus with at least one additional ASCVD risk factor, who had elevated fasting triglyceride levels $(\geq$ $150 \mathrm{mg} / \mathrm{dL}$ and $<500 \mathrm{mg} / \mathrm{dL}$ ) and relatively well-controlled LDL-C $(>40 \mathrm{mg} / \mathrm{dL}$ and $\leq 100 \mathrm{mg} / \mathrm{dL})$. Treatment with icosapent ethyl was previously demonstrated to significantly reduce of primary endpoint events (composite of cardiovascular death, nonfatal MI, nonfatal stroke, coronary revascularization, or unstable angina requiring hospitalization) (HR 0.75 ; 95\% CI, 0.68-0.83; $p<0.001 ; \mathrm{ARR}=4.8 \%$ ) over a median follow-up of 4.9 years [22•, 23, 24].

\section{Study Overview}

REDUCE-IT EPA assessed the association between baseline and achieved serum EPA levels with clinical outcomes [25]. Median levels of EPA in the icosapent ethyl group and placebo group were both $26.1 \mu \mathrm{g} / \mathrm{mL}$ at baseline. Median EPA levels at year 1 and year 5 were $144 \mu \mathrm{g} / \mathrm{mL}$ and $158 \mu \mathrm{g} / \mathrm{mL}$ in the icosapent ethyl group versus $23.3 \mu \mathrm{g} / \mathrm{mL}$ and $25.3 \mu \mathrm{g} /$ $\mathrm{mL}$ in the placebo group. On-treatment daily average for the absolute change in EPA level from baseline between icosapent ethyl and placebo groups was $103.8 \mu \mathrm{g} / \mathrm{mL}$ and was $247.7 \%$ for percent change, $p<0.0001$. Meanwhile, the levels of docosahexaenoic acid (DHA) decreased by $2.9 \%$. The study showed that there was no heterogeneity of effect on the primary composite endpoint with respect to baseline EPA levels stratified by tertiles ( $p$ interaction 0.91 ).

Next, the impact of select biomarkers (EPA, triglycerides, LDL-C, HDL-C, non-HDL-C, apoB, high sensitivity Creactive protein, remnant like protein cholesterol) on the effect of icosapent ethyl on primary composite endpoint events was assessed by adjustment of each biomarker as a time-varying covariate. This analysis suggests that the effect of icosapent ethyl on events is independent of triglycerides and other biomarkers evaluated except EPA - the effect of icosapent ethyl on outcomes was attenuated after adjustment for EPA level (HR 1.03, 95\% CI 0.91-1.16 after adjustment). Finally, the study showed that there was a dose-dependent effect of ontreatment EPA levels with primary composite outcome events as well as individual components of the composite outcome with high EPA levels corresponding to higher risk reduction for events $(p<0.001$ for all). There was further a dosedependent effect of on-treatment EPA level with sudden cardiac death, cardiac arrest and heart failure outcomes $(p<0.001$ for all).

\section{Clinical Implications}

The REDUCE-IT trial demonstrated the ability of icosapent ethyl to significantly reduce ASCVD risk in secondary 
prevention and high-risk primary prevention patients with elevated triglycerides but relatively well-controlled LDL-C on stable statin therapy. While elevated triglyceride levels likely represent a marker of residual risk of ASCVD, the degree of ASCVD risk reduction observed in trial cannot be fully explained by a reduction in triglycerides. The REDUCE-IT EPA analysis suggest that the clinical benefit of icosapent ethyl likely depends on the serum EPA level achieved while ontreatment. Further studies are needed to explore whether the association between achieved EPA and ASCVD risk reduction can be extended beyond treatment with icosapent ethyl. Increasing serum EPA may be a potential mechanism by which to target residual risk for ASCVD beyond LDL-C lowering.

\section{Integrating the Effect of Polygenic Scores, Low Density Lipoproteins, and Systolic Blood Pressure on the Lifetime Risk of Cardiovascular Disease}

While some disease such as HoFH are caused by mutations at a single gene, most cardiovascular diseases are a result of complex interactions of multiple genes in combination with environmental factors. Polygenic risk scores (PGS) estimate the summative risk of genetic variants within the genome that may contribute to phenotypic manifestation of a disease [26]. Since each individual's genetic makeup is largely set at birth, PGS have the potential to estimate the contribution of one's genetic variations towards lifetime risk of disease even at an early age. However, PGS is not deterministic and contributions from modifiable risk factors over time influences the development of disease.

\section{Study Overview}

This study assessed how differences in lifetime exposure of LDL-C and SBP affected lifetime risk for coronary artery disease (CAD) across categories of PGS and included 445,566 participants from the UK Biobank [27]. The mean age at enrollment was 57.2 years, and $54 \%$ of those enrolled were women. Mean LDL-C for the cohort was $138 \mathrm{mg} / \mathrm{dL}$, while the mean SBP was $137.8 \mathrm{mmHg}$. The PGS for CAD was constructed based on 4,037,825 genetic variants after exclusion of variants with significant association with LDL or SBP. Meanwhile, variants with significant association with LDL-C or SBP were used to construct Mendelian Randomization instruments to estimate lifetime exposure to these risk factors. The primary outcome was first occurrence of a major coronary event (fatal or nonfatal myocardial infarction or coronary revascularization).

In continuous analysis, PGS was significantly associated with major coronary events (HR 1.45, 95\% CI $1.43-$
1.47). A stepwise increase in risk for coronary events was further observed across increasing quintiles of PGS where quintile 1 (lowest) was treated as the reference group (quintile 5 vs. quintile 1: HR 2.77, 95\% CI 2.65-2.89). Lifetime exposure of LDL-C and SBP appeared to significantly modify the effect of PGS on cumulative lifetime risk of major coronary events across all PGS quintiles. For instance, lifetime CV risk was significantly higher in individuals with the lowest PGS but with high exposure (+ $1 \mathrm{mmol}[38.7 \mathrm{mg} / \mathrm{dL}] \mathrm{LDL}$ and $10 \mathrm{mmHg}$ of the population mean) to LDL-C and SBP compared with those in the highest PGS quintile with low exposure $(-1 \mathrm{mmol}$ [38.7 $\mathrm{mg} / \mathrm{dL}$ ] LDL-C and $10 \mathrm{mmHg}$ ) to LDL-C and SBP $(p<0.001)$. Those with low lifetime exposure to LDL-C and SBP had consistently low lifetime risk for CAD across PGS quintiles. Not surprisingly, individuals with high PGS and high exposure to LDL-C and SBP derived the most benefit from LDL-C lowering (ARR $13.02 \%$ ). Individuals with high PGS but with intermediate or low exposure to LDL-C and SBP had only modest lifetime risk reduction with LDL-C lowering (ARR $3.11 \%$ and ARR $0.69 \%$, respectively).

\section{Clinical Implications}

The results of this study show that while the PGS estimates one's lifetime risk for $\mathrm{CAD}$, the trajectory of disease development is significantly influenced by modifiable risk factors such as LDL-C and SBP. Therefore, PGS should be viewed in the context of other risk factors. The use of PGS in the clinical setting has thus far been controversial. Prior studies have suggested that its use did not significantly enhance the discrimination or risk reclassification when added to traditional clinical risk predictors for ASCVD [28]. One area in which PGS may be valuable is in the risk assessment of ASCVD in younger patients when traditional risk stratification tools such as the pooled cohort equation are not well validated. PGS in this setting, when combined with screening of lipids and blood pressure among other clinical risk factors, can potentially help guide clinicians in when and how aggressively to initiate therapeutic interventions in order to mitigate lifetime risk for ASCVD. This may also motivate patients to implement positive therapeutic lifestyle changes early on. However, large epidemiology studies are needed to validate the risk prediction utility of PGS at younger age.

\section{Conclusion}

The studies discussed above, which were presented at ACC.20/WCC, represent a sample of the continuing innovations made in the field of CVD prevention. More efforts are 
needed to validate these novel strategies in the identification and treatment of CVD risk factors, in order to translate these study findings towards improving patient care delivery in the real-world setting.

\section{Compliance with Ethical Standards}

Conflict of Interest Dr. Virani reports grants from Department of Veterans Affairs, World Heart Federation, Tahir and Jooma Family, other from American College of Cardiology, other from Steering Committee Member, outside the submitted work.

Dr. Jia has nothing to disclose.

Dr. Al Rifai has nothing to disclose.

Dr. Liu has nothing to disclose.

Dr. Agrawala has nothing to disclose.

Dr. Gulati has nothing to disclose.

Human/Animal Rights Statement This article does not contain any studies with human or animal subjects performed by any of the authors.

\section{References}

Papers of particular interest, published recently, have been highlighted as:

- Of importance

•- Of major importance

1. Jia X, Al Rifai M, Gluckman TJ, Birnbaum Y, Virani SS. Highlights from selected cardiovascular disease prevention studies presented at the 2019 European Society of Cardiology Congress. Curr Atheroscler Rep. 2019;21(12):46.

2. Al Rifai M, Jia X, Al-Mallah MH, Miedema MD, Martin SS, Virani SS. Major randomized clinical trials in cardiovascular disease prevention presented at the 2019 American College of Cardiology Annual Scientific Session. Curr Atheroscler Rep. 2019;21(8):31.

3. Mehta A, Mahtta D, Gulati M, Sperling LS, Blumenthal RS, Virani SS. Cardiovascular disease prevention in focus: highlights from the 2019 American Heart Association Scientific Sessions. Curr Atheroscler Rep. 2020;22(1):3.

4. Soutar AK, Naoumova RP. Mechanisms of disease: genetic causes of familial hypercholesterolemia. Nat Clin Pract Cardiovasc Med. 2007;4(4):214-25.

5. Brautbar A, Leary E, Rasmussen K, Wilson DP, Steiner RD, Virani S. Genetics of familial hypercholesterolemia. Curr Atheroscler Rep. 2015;17(4):491.

6. Bandeali SJ, Daye J, Virani SS. Novel therapies for treating familial hypercholesterolemia. Curr Atheroscler Rep. 2014;16(1):382.

7. Cuchel M, Bruckert E, Ginsberg HN, Raal FJ, Santos RD, Hegele RA, et al. Homozygous familial hypercholesterolaemia: new insights and guidance for clinicians to improve detection and clinical management. A position paper from the Consensus Panel on Familial Hypercholesterolaemia of the European Atherosclerosis Society. Eur Heart J. 2014;35(32):2146-57.

8. Ito MK, Watts GF. Challenges in the diagnosis and treatment of homozygous familial hypercholesterolemia. Drugs. 2015;75(15): 1715-24.

9.• Schwartz GG, Steg PG, Szarek M, Bhatt DL, Bittner VA, Diaz R, et al. Alirocumab and cardiovascular outcomes after acute coronary syndrome. N Engl J Med. 2018;379(22):2097-107 Large, randomized, placebo-controlled trial assessing the efficacy and safety of alirocumab in risk reduction of ASCVD outcomes.

10. Blom D, editor Alirocumab efficacy and safety in adults with homozygous familial hypercholesterolemia (ODYSSEY HoFH). American College of Cardiology 2020 Scientific Session; March 30, 2020.

11. Dewey FE, Gusarova V, Dunbar RL, O'Dushlaine C, Schurmann $\mathrm{C}$, Gottesman O, et al. Genetic and pharmacologic inactivation of ANGPTL3 and cardiovascular disease. N Engl J Med. 2017;377(3): 211-21.

12. Wang X, Musunuru K. Angiopoietin-like 3: from discovery to therapeutic gene editing. JACC Basic Transl Sci. 2019;4(6):755-62.

13. Gaudet D, Gipe DA, Pordy R, Ahmad Z, Cuchel M, Shah PK, et al. ANGPTL3 inhibition in homozygous familial hypercholesterolemia. N Engl J Med. 2017;377(3):296-7.

14. Raal F, editor Evinacumab in Patients with Homozygous Familial Hypercholesterolemia. American College of Cardiology 2020 Scientific Session; March 30, 2020.

15. Raal FJ, Hovingh GK, Blom D, Santos RD, Harada-Shiba M, Bruckert E, et al. Long-term treatment with evolocumab added to conventional drug therapy, with or without apheresis, in patients with homozygous familial hypercholesterolaemia: an interim subset analysis of the open-label TAUSSIG study. Lancet Diabetes Endocrinol. 2017;5(4):280-90 Evaluation of the efficacy and safety of evolocumab in the reduction of LDL-C in a subset of patients with HoFH enrolled in the open-labeled, nonrandomized phase III trial.

16. Eisenberg $\mathrm{M}$, editor $\mathrm{A}$ randomized controlled trial evaluating the efficacy and safety of e-cigarettes for smoking cessation. American College of Cardiology 2020 Scientific Session; March 30, 2020.

17. Böhm M, Kario K, Kandzari DE, Mahfoud F, Weber MA, Schmieder RE, et al. Efficacy of catheter-based renal denervation in the absence of antihypertensive medications (SPYRAL HTNOFF MED Pivotal): a multicentre, randomised, sham-controlled trial. Lancet. 2020;395:1444-51.

18. Armstrong PW, Pieske B, Anstrom KJ, Ezekowitz J, Hernandez $\mathrm{AF}$, Butler $\mathrm{J}$, et al. Vericiguat in patients with heart failure and reduced ejection fraction. N Engl J Med. 2020.

19. Ray KK, Wright RS, Kallend D, Koenig W, Leiter LA, Raal FJ, et al. Two phase 3 trials of inclisiran in patients with elevated LDL cholesterol. N Engl J Med. 2020; Two phase III trials on the efficacy and safety of inclisiran in the reduction of LDL-C in patients with ASCVD or ASCVD risk equivalent.

20. Raal FJ, Kallend D, Ray KK, Turner T, Koenig W, Wright RS, et al. Inclisiran for the treatment of heterozygous familial hypercholesterolemia. N Engl J Med. 2020. Phase III trial on the efficacy and safety of inclisiran in the reduction of LDL-C in patients with heterozygous familial hypercholesterolemia.

21. Wright RS, editor ORION: A pooled analysis of phase III studies of inclisiran. American College of Cardiology 2020 Scientific Session; March 30, 2020.

22.• Bhatt DL, Steg G, Miller M, Brinton EA, Jacobson TA, Ketchum $\mathrm{SB}$, et al. Cardiovascular risk reduction with icosapent ethyl for hypertriglyceridemia. New England Journal of Medicine. 2019;380(1):11-22 Large, randomized, placebo-controlled cardiovascular outcome trial of icosapent ethyl assessing efficacy and safety in the reduction of ASCVD risk.

23. Jia X, Kohli P, Virani SS. Omega-3 fatty acid and cardiovascular outcomes: insights from recent clinical trials. Curr Atheroscler Rep. 2019;21(1):1.

24. Jia X, Koh S, Al Rifai M, Blumenthal RS, Virani SS. Spotlight on icosapent ethyl for cardiovascular risk reduction: evidence to date. Vasc Health Risk Manag. 2020;16:1-10.

25. Bhatt DL, editor EPA levels and cardiovascular outcomes in the reduction of cardiovascular events with icosapent ethyl-intervention 
trial. American College of Cardiology 2020 Scientific Session; March 30, 2020.

26. Sugrue LP, Desikan RS. What are polygenic scores and why are they important? JAMA. 2019;321(18):1820-1.

27. Ference BA, editor Integrating the effect of polygenic scores, low density lipoproteins and systolic blood pressure on the lifetime risk of cardiovascular disease. American College of Cardiology 2020 Scientific Session; March 28, 2020.
28. Mosley JD, Gupta DK, Tan J, Yao J, Wells QS, Shaffer CM, et al. Predictive accuracy of a polygenic risk score compared with a clinical risk score for incident coronary heart disease. JAMA. 2020;323(7):627-35.

Publisher's Note Springer Nature remains neutral with regard to jurisdictional claims in published maps and institutional affiliations. 